\title{
EXAMPLES OF COORBIT SPACES FOR DUAL PAIRS
}

\author{
JENS GERLACH CHRISTENSEN AND GESTUR ÓLAFSSON
}

\begin{abstract}
In this paper we summarize and give examples of a generalization of the coorbit space theory initiated in the 1980's by H.G. Feichtinger and K.H. Gröchenig. Coorbit theory has been a powerful tool in characterizing Banach spaces of distributions with the use of integrable representations of locally compact groups. Examples are a wavelet characterization of the Besov spaces and a characterization of some Bergman spaces by the discrete series representation of $\mathrm{SL}_{2}(\mathbb{R})$. We present examples of Banach spaces which could not be covered by the previous theory, and we also provide atomic decompositions for an example related to a non-integrable representation.
\end{abstract}

October 26, 2018

\section{INTRODUCTION}

In the 1980's H. G. Feichtinger and K. H. Gröchenig developed a unified theory for construction of Banach spaces and atomic decompositions using representation theory (see [4, 5, 6]). The construction, called coorbit space theory, relies on an irreducible unitary representation $(\pi, \mathcal{H})$ of a locally compact Hausdorff group $G$ with left invariant Haar measure $d x$. A further requirement is that $\pi$ is integrable, in other words it is required that there exists a non-zero $u \in \mathcal{H}$ such that

$$
\int_{G}\left|(\pi(x) u, u)_{\mathcal{H}}\right| d x<\infty
$$

Other constructions carry similar features as the theory of Feichtinger and Gröchenig, though they are not related to an integrable nor irreducible representation. For example the Banach spaces of band-limited functions on a locally compact abelian group $G$ in [8], are related to the regular representation $\ell$ on $L^{2}(G)$. This representation is neither irreducible nor integrable (for non-trivial $G$ ) and thus the spaces of band-limited function cannot be described as coorbit spaces. In [4, Section 7.3] it is also suggested that coorbit spaces related to nonintegrable representations seem possible. The authors have developed a more general theory in [2], which does neither require integrability nor irreducibility. In the present article we give a short description of this theory, but the main aim is to give several examples explaining the general construction. Most of these examples are not covered by the theory of Feichtinger and Gröchenig.

In section 2 we present our generalization of the coorbit space theory of Feichtinger and Gröchenig. Next we show why smooth representations will make the construction easier and we conclude the article with examples, some of which illustrate the use of the generalized

2000 Mathematics Subject Classification. Primary 43A15,42B35; Secondary 22D12.

Key words and phrases. Coorbit spaces, Gelfand Triples, Representation theory of Locally Compact Groups.

The first author gratefully acknowledges support from the Louisiana Board of Regents under grant LEQSF(2005-2007)-ENH-TR-21 and NSF grant DMS-0801010.

The research of the second author was supported by NSF grants DMS-0402068 and DMS-0801010. 
coorbit space theory. The examples include coorbit spaces for Sobolev spaces on the $(a x+$ b)-group, Bergman spaces and discrete series representation of $\mathrm{SL}_{2}(\mathbb{R})$, and bandlimited functions on a Gelfand pair $(G, K)$ where $K$ is compact.

\section{Generalized Coorbit spaces}

In this section we will suggest a minimal set of requirements for the construction of general coorbit spaces. This minimal set of requirements will allow us to lift the restriction of only working with integrable representations, and instead work with the bigger class of square integrable representations and also non-irreducible representations. Most proofs have been omitted, and we refer the reader to [2] for the details.

Let $S$ be a Fréchet space and let $S^{*}$ be the space of continuous conjugate linear functionals on $S$ equipped with the weak topology. We assume that $S$ is continuously imbedded and weakly dense in $S^{*}$. The conjugate dual pairing of elements $v \in S$ and $v^{\prime} \in S^{*}$ will be denoted by $\left\langle v^{\prime}, v\right\rangle$.

Let $G$ be a locally compact group with a fixed left Haar measure $d x$, and assume that $(\pi, S)$ is a representation of $G$. Also assume that the representation is continous,i.e. $g \mapsto \pi(g) v$ is continuous for all $v \in S$. As usual define the contragradient representation $\left(\pi^{*}, S^{*}\right)$ by

$$
\left\langle\pi^{*}(x) v^{\prime}, v\right\rangle=\left\langle v^{\prime}, \pi\left(x^{-1}\right) v\right\rangle .
$$

Then $\pi^{*}$ is a continuous representation of $G$ on $S^{*}$. For a fixed vector $u \in S$ define the linear map $V_{u}: S^{*} \rightarrow C(G)$ by

$$
V_{u}\left(v^{\prime}\right)(x)=\left\langle v^{\prime}, \pi(x) u\right\rangle
$$

The map $V_{u}$ is called the voice transform or the wavelet transform.

Assumption 2.1. Let $Y$ be a left invariant Banach Space of Functions on $G$, and assume that there is a non-zero cyclic vector $u \in S$ satisfying the following properties

(R1) the reproducing formula $V_{u}(v) * V_{u}(u)=V_{u}(v)$ is true for all $v \in S$

(R2) the space $Y$ is stable under convolution with $V_{u}(u)$ and $f \mapsto f * V_{u}(u)$ is continuous

(R3) if $f=f * V_{u}(u) \in Y$ then the mapping $S \ni v \mapsto \int f(x)\left\langle\pi^{*}(x) u, v\right\rangle d x \in \mathbb{C}$ is in $S^{*}$

(R4) the mapping $S^{*} \ni v^{\prime} \mapsto \int\left\langle v^{\prime}, \pi(x) u\right\rangle\left\langle\pi^{*}(x) u, u\right\rangle d x \in \mathbb{C}$ is weakly continuous

A vector $u$ satisfying Assumption 2.1] is called an analyzing vector. Note that (R4) implies that there is an element $v \in S$ such that

$$
\left\langle v^{\prime}, v\right\rangle=\int\left\langle v^{\prime}, \pi(x) u\right\rangle\left\langle\pi^{*}(x) u, u\right\rangle d x
$$

for all $v^{\prime} \in S^{*}$. If we use the notation $f^{\vee}(x)=f\left(x^{-1}\right)$ then $v=\pi\left(V_{u}(u)^{\vee}\right) u$.

Theorem 2.2. Assume that $Y$ and $u$ satisfy Assumption 2.1 and define the coorbit space

$$
\operatorname{Co}_{S}^{u} Y=\left\{v^{\prime} \in S^{*} \mid V_{u}\left(v^{\prime}\right) \in Y\right\}
$$

equipped with the norm $\left\|v^{\prime}\right\|=\left\|V_{u}\left(v^{\prime}\right)\right\|_{Y}$. Then the following properties hold

(a) $V_{u}(v) * V_{u}(u)=V_{u}(v)$ for $v \in \operatorname{Co}_{S}^{u} Y$.

(b) The space $\operatorname{Co}_{S}^{u} Y$ is a $\pi^{*}$-invariant Banach space.

(c) $V_{u}: \operatorname{Co}_{S}^{u} Y \rightarrow Y$ intertwines $\pi^{*}$ and left translation

(d) The convolution operator $f \mapsto f * V_{u}$ is a bounded projection from $Y$ to the closed subspace $V_{u}\left(\operatorname{Co}_{S}^{u} Y\right)=Y * V_{u}(u)$.

(e) $\operatorname{Co}_{S}^{u} Y=\left\{\pi^{*}\left(f * V_{u}(u)\right) u \mid f \in Y\right\}$. 
(f) $V_{u}: \mathrm{Co}_{S}^{u} Y \rightarrow Y * V_{u}(u)$ is an isometric isomorphism

The following theorem tells us which analyzing vectors will give the same coorbit space.

Theorem 2.3. If $u_{1}$ and $u_{2}$ both satisfy Assumption 2.1 and for $i, j \in\{1,2\}$ the following properties can be verified

- there are non-zero constants $c_{i, j}$ such that $V_{u_{i}}(v) * V_{u_{j}}\left(u_{i}\right)=c_{i, j} V_{u_{j}}(v)$ for all $v \in S$

- $Y \ni f \mapsto f * V_{u_{i}}\left(u_{j}\right) \in Y$ is continuous

- $S^{*} \ni v^{\prime} \mapsto \int\left\langle v^{\prime}, \pi(x) u_{i}\right\rangle\left\langle\pi^{*}(x) u_{i}, u_{j}\right\rangle d x \in \mathbb{C}$ is weakly continuous

then $\operatorname{Co}_{S}^{u_{1}} Y=\operatorname{Co}_{S}^{u_{2}} Y$ with equivalent norms.

In the following we will describe how the choice of the Fréchet space $S$ affects the coorbit space. We will show that there is great freedom when choosing $S$.

Theorem 2.4. Let $S$ and $T$ be Fréchet spaces which are weakly dense in their conjugate duals $S^{*}$ and $T^{*}$ respectively. Let $\pi$ and $\tilde{\pi}$ denote representations of $G$ on $S$ and $T$ respectively. Assume there is a vector $u \in S$ and $\widetilde{u} \in T$ such that the requirements in Assumption 2.1 are satisfied by both $(u, S)$ and $(\widetilde{u}, T)$. Also assume that the conjugate dual pairings of $S^{*} \times S$ and $T^{*} \times T$ satisfy $\langle u, \pi(x) u\rangle_{S}=\langle\widetilde{u}, \widetilde{\pi}(x) \widetilde{u}\rangle_{T}$ for all $x \in G$. Then $\operatorname{Co}_{S}^{u} Y$ and $\operatorname{Co}_{T}^{\widetilde{u}} Y$ are isometrically isomorphic. The isomorphism is given by $V_{\widetilde{u}} V_{u}^{-1}$.

Let $\pi$ be a unitary irreducible representation of $G$ on $\mathcal{H}$. Assume that the Fréchet spaces $S$ and $T$ are $\pi$-invariant and that $\left(S, \mathcal{H}, S^{*}\right)$ and $\left(T, \mathcal{H}, T^{*}\right)$ are Gelfand triples with the common Hilbert space $\mathcal{H}$. Then $S \cap T$ is $\pi$-invariant and if we can pick a non-zero vector $u \in S \cap T$, such that $u$ is analyzing for both $S$ and $T$, then

$$
\langle u, \pi(x) u\rangle_{S}=(u, \pi(x) u)_{\mathcal{H}}=\langle u, \pi(x) u\rangle_{T}
$$

and we are in the situation of the previous theorem. We summarize the statement as

Corollary 2.5. Assume that $\left(S, \mathcal{H}, S^{*}\right)$ and $\left(T, \mathcal{H}, T^{*}\right)$ are Gelfand tripples and assume there is an analyzing vector $u \in S \cap T$ such that both $(u, S)$ and $(u, T)$ satisfy Assumption 2.1 for some Banach space $Y$, then $\operatorname{Co}_{S}^{u} Y$ and $\operatorname{Co}_{T}^{u} Y$ are isometrically isomorphic.

If the Fréchet space $S$ is a dense subspace of the Fréchet space $T$, and $S$ is continuously included in $T$, then we can regard the space $T^{*}$ as a subspace of $S^{*}$. With this identification the two coorbit spaces will be equal. We state the following

Theorem 2.6. Let $(\pi, \mathcal{H})$ be a unitary irreducible representation of $G$, and let $\left(S, \mathcal{H}, S^{*}\right)$ and $\left(T, \mathcal{H}, T^{*}\right)$ be Gelfand triples for which $(\pi, S)$ and $(\pi, T)$ are representations of $G$. Assume that $i: S \rightarrow T$ is a continuous linear inclusion and that there is $u \in S$ such that both $(u, S)$ and $(i(u), T)$ satisfy Assumption 2.1. Then the map $i^{*}$ restricted to $\operatorname{Co}_{T}^{i(u)} Y$ is an isometric isomorphism between $\operatorname{Co}_{T}^{i(u)} Y$ and $\operatorname{Co}_{S}^{u} Y$.

Remark 2.7. If $(\pi, S)$ is a representation of $G$ and $u$ is a cyclic vector for which it is true

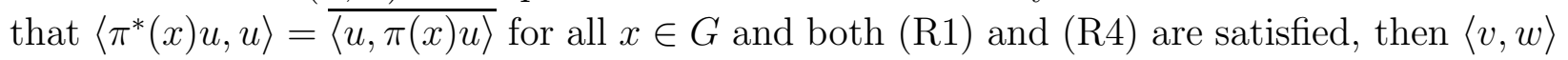
is an inner product on $S$. The completion $\mathcal{H}$ of $S$ with respect to the norm $\|v\|_{\mathcal{H}}=\sqrt{\langle v, v\rangle}$ is a Hilbert space. The representation $\pi$ will then extend to a unitary representation $\widetilde{\pi}$ on $\mathcal{H}$, but we will not be able to conclude that $\tilde{\pi}$ is irreducible. Therefore the construction of coorbit spaces also works for non-irreducible representations, as long as we choose a cyclic vector in the Fréchet space $S$.

Note also that a reproducing formula has been constructed from a non-unitary representation in [23], thus allowing for construction of coorbit spaces in this new setting. 


\section{SMOOTH SQUARE INTEGRABLE REPRESENTATIONS}

We will now point out why smooth representations are easy to work with. Let $(\pi, \mathcal{H})$ be a square integrable representation of a Lie group $G$. This means that $\pi$ is unitary and irreducible and that there is a non-zero $u$ such that

$$
\int_{G}\left|(u, \pi(x) u)_{\mathcal{H}}\right|^{2} d x<\infty
$$

For square integrable representations the following theorem found in [3] is crucial

Theorem 3.1 (Duflo-Moore). If $(\pi, \mathcal{H})$ is square integrable, then there is a positive densely defined operator $C$ with domain $D(C)$ such that $\langle u, \pi(\cdot) u\rangle$ is in $L^{2}(G)$ if and only if $u \in D(C)$. Furthermore, if $u_{1}, u_{2} \in D(C)$ then

$$
\int_{G}\left(v_{1}, \pi(x) u_{1}\right)_{\mathcal{H}}\left(\pi(x) u_{2}, v_{2}\right)_{\mathcal{H}} d x=\left(C u_{2}, C u_{1}\right)_{\mathcal{H}}\left(v_{1}, v_{2}\right)_{\mathcal{H}}
$$

Picking $u$ such that $\|C u\|=1$ we are able to obtain the reproducing formula

$$
V_{u}(v) * V_{u}(u)=V_{u}(v)
$$

for $v \in \mathcal{H}$. Denote the space of smooth vectors in $\mathcal{H}$ by $\mathcal{H}_{\pi}^{\infty}$ equipped with the usual Fréchet space topology (see [21, p. 253]). Denote by $\mathcal{H}_{\pi}^{-\infty}$ the conjugate dual of $\mathcal{H}_{\pi}^{\infty}$. A vector $\pi(f) v=\int f(x) \pi(x) v d x$ for $f \in C_{c}^{\infty}(G)$ and $v \in \mathcal{H}$ is called a Gårding vector. In the following we let the Fréchet space $S$ be $\mathcal{H}_{\pi}^{\infty}$. The reproducing formula (2) is then true for $v \in \mathcal{H}_{\pi}^{\infty}$, and the following result is used to extend it to $v \in \mathcal{H}_{\pi}^{-\infty}$.

Proposition 3.2. If $u \in \mathcal{H}_{\pi}^{\infty}$ is in the domain of the operator $C$ from Theorem [3.1, then the map

$$
\mathcal{H}_{\pi}^{-\infty} \ni v^{\prime} \mapsto \int\left\langle v^{\prime}, \pi(x) u\right\rangle\langle\pi(x) u, u\rangle d x \in \mathbb{C}
$$

is continuous in the weak topology, and thus ( $(\mathrm{R} 4)$ is satisfied.

The next two results state, that if there is a non-zero $u \in \mathcal{H}$ satisfying (R1) and (R2), then there is a smooth non-zero vector which satisfies the same conditions.

Proposition 3.3. Let $f \in C_{c}^{\infty}(G)$ then $\pi(f) D(C) \subseteq D(C)$. If $u \in \mathcal{H}$ satisfies (R1), then so will a constant multiple of $\pi(f) u$.

Proof. Assume that $u \in D(C)$ and $f \in C_{c}^{\infty}(G)$. Then

$$
\begin{aligned}
V_{\pi(f) u}(\pi(f) u)(z) & =\iint f(x) f(y)(\pi(y) u, \pi(z x) u)_{\mathcal{H}} d x d y \\
& =f * V_{u}(u) *\left(f^{\vee}\right)(z)
\end{aligned}
$$

where $f^{\vee}(x)=f\left(x^{-1}\right)$. Since $f, f^{\vee} \in C_{c}(G) \subseteq L^{1}(G)$ and $V_{u}(u) \in L^{2}(G)$, the function $f * V_{u}(u) * f^{\vee}$ is in $L^{2}(G)$. This shows that $\pi(f) u$ is in the domain of $C$.

A Banach Function space $Y$ is called solid if $|f| \leq|g|$ a.e. and $g \in Y$ imply that $f \in Y$.

Proposition 3.4. Assume that $Y$ is a solid Banach Function space, and that $Y \ni F \mapsto$ $F * g \in Y$ is continuous for each $g \in C_{c}^{\infty}(G)$. Further assume that there is a $u \in \mathcal{H}$ such that $Y \ni F \mapsto F *\left|V_{u}(u)\right| \in Y$ is continuous. Then any Gårding vector of the form $\pi(f) u$ satisfies (ㅈ2). 
Remark 3.5. Similar results can be shown for the space $\mathcal{H}_{\pi}^{\omega}$ of analytic vectors, and its dual $\mathcal{H}_{\pi}^{-\omega}$, by approximating the Gårding vector $\pi(f) u$ by an analytic vector cf. [21, p. 278 ff.].

With these results in place the Gelfand triples $\left(\mathcal{H}_{\pi}^{\infty}, \mathcal{H}, \mathcal{H}_{\pi}^{-\infty}\right)$ and $\left(\mathcal{H}_{\pi}^{\omega}, \mathcal{H}, \mathcal{H}_{\pi}^{-\omega}\right)$ will be natural choices for the construction of some coorbit spaces.

\section{EXAMPLES}

In this section we will give some examples of coorbit spaces and also show that the generalization presented in this paper is in fact a generalization of the theory by Feichtinger and Gröchenig.

4.1. Coorbit spaces by Feichtinger and Gröchenig. Here we will show that the coorbit spaces of Feichtinger and Gröchenig are special cases of the general coorbit spaces. In the theory of Feichtinger and Gröchenig (see [4, 5, 6] the Banach Function Space $Y$ is assumed to be left- and right-invariant and also a right $L_{m}^{1}(G)$-module for some submultiplicative weight $m$ satisfying $m(x) \geq 1$. It is assumed that

$$
\|f * g\|_{Y} \leq\|f\|_{Y}\|g\|_{L_{m}^{1}}
$$

Let $(\pi, \mathcal{H})$ be a square-integrable irreducible unitary representation and let

$$
V_{u}(v)(x)=(v, \pi(x) u)_{\mathcal{H}}
$$

and assume that

$$
A_{m}=\left\{u \in \mathcal{H} \mid V_{u}(u) \in L_{m}^{1}(G)\right\}
$$

is not trivial. Then we can pick a non-zero $u \in A_{m}$ and define

$$
\mathcal{H}_{m}^{1}=\left\{u \in \mathcal{H} \mid V_{u}(v) \in L_{m}^{1}(G)\right\}
$$

equipped with the norm $\|v\|_{\mathcal{H}_{m}^{1}}=\left\|V_{u}(v)\right\|_{L_{m}^{1}}$. In [4, Lemma 4.2] Feichtinger and Gröchenig show that $\mathcal{H}_{m}^{1}$ is a $\pi$-invariant Banach space independent of the chosen $u \in A_{m}$ (where different $u$ give equivalent norms). They also show that $\left(\pi, \mathcal{H}_{w}^{1}\right)$ is irreducible and therefore every vector $u \in A_{w}$ is cyclic. These assumptions ensure that (R1) and (R2) are automatically satisfied as has been proven in [5, Theorem 4.1]. By the same theorem the conjugate dual $\left(\mathcal{H}_{m}^{1}\right)^{*}$ of $\mathcal{H}_{m}^{1}$ consists of linear functionals $v^{\prime}$ for which $V_{u}\left(v^{\prime}\right) \in L_{1 / m}^{\infty}(G)$, and thus also (R4) is satisfied. It requires some work (see [5, Proposition 4.3]) to show that if $f \in Y$ satisfies $f=f * V_{u}(u)$ then $f \in L_{1 / m}^{\infty}(G)$ and thus $\pi(f) u$ defined by

$$
\langle\pi(f) u, v\rangle=\int f(x)\langle\pi(x) u, v\rangle d x
$$

is an element in $\left(\mathcal{H}_{w}^{1}\right)^{*}$. Therefore also $(\underline{\mathrm{R} 3})$ is satisfied and the coorbit space CoY of Feichtinger and Gröchenig is the space

$$
\mathrm{Co} Y=\operatorname{Co}_{\mathcal{H}_{m}^{1}}^{u} Y=\left\{v^{\prime} \in\left(\mathcal{H}_{w}^{1}\right)^{*} \mid V_{u}\left(v^{\prime}\right) \in Y\right\}
$$

with norm

$$
\left\|v^{\prime}\right\|_{\mathrm{Co} Y}=\left\|V_{u}\left(v^{\prime}\right)\right\|_{Y}
$$

The independence of $u$ follows from the construction of $\mathcal{H}_{m}^{1}$ and Theorem 2.3 . 
4.2. Homogeneous Besov Spaces. We will now go through the calculations showing that some homogeneous Besov spaces can be described as coorbits. This gives a wavelet decomposition of these spaces. The example has already been covered in [4, 15, 18].

The non-connected $(a x+b)$-group $G$ is the semidirect product $\mathbb{R}^{*} \ltimes \mathbb{R}$, i.e. the set $\mathbb{R}^{*} \times \mathbb{R}$ with group composition $(a, b)\left(a^{\prime}, b^{\prime}\right)=\left(a a^{\prime}, a b^{\prime}+b\right)$. The left Haar measure on the $a x+b$ group is given by

$$
f \mapsto \int_{\mathbb{R}} \int_{\mathbb{R}^{*}} f(a, b) \frac{d a d b}{a^{2}}
$$

for $f \in C_{c}(G)$, where $d a$ and $d b$ are Lebesgue measures on $\mathbb{R}$. The representation

$$
\pi(a, b) v(t)=\frac{1}{\sqrt{|a|}} v\left(\frac{t-b}{a}\right)
$$

is an irreducible unitary representation of $G$ on $\mathcal{H}=L^{2}(\mathbb{R})$. In the Fourier picture $\pi$ has the form

$$
\widehat{\pi}(a, b) \widehat{v}(t)=e^{i b t} \sqrt{|a|} \widehat{v}(a t)
$$

Let $\mathcal{S}(\mathbb{R})$ be the space of rapidly decreasing smooth functions with the usual topology, and let $\mathcal{S}_{0}$ be the space

$$
\mathcal{S}_{0}=\left\{v \in \mathcal{S}(\mathbb{R}) \mid \int x^{n} v(x) d x=i D^{n} \widehat{v}(0)=0\right\}
$$

with the subspace topology. $\mathcal{S}_{0}$ is a $\pi$-invariant Fréchet space. The conjugate dual space $\mathcal{S}_{0}^{*}$ is isomorphic to

$$
\mathcal{S}_{0}^{*} \cong \mathcal{S}^{\prime}(\mathbb{R}) /\left\{\Lambda_{P} \mid P \text { is a polynomial }\right\}
$$

where $\Lambda_{P}$ is the functional given by $\Lambda_{P}(v)=\int P(x) v(x) d x$ for $v \in \mathcal{S}(\mathbb{R})$. Denote the conjugate dual pairing by $\left\langle v^{\prime}, v\right\rangle$ (which extends the inner product on $L^{2}(\mathbb{R})$ ) and the voice transform

$$
V_{v}\left(v^{\prime}\right)(x)=\left\langle v^{\prime}, \pi(x) v\right\rangle
$$

for $v \in \mathcal{S}_{0}$ and $v^{\prime} \in \mathcal{S}_{0}^{\prime}$.

It is known (see [12, Theorem 14.0.2, p. 66]) that if

$$
0<\int_{-\infty}^{\infty} \frac{|\widehat{u}(x)|^{2}}{|x|} d x<\infty
$$

then for any $v \in \mathcal{S}_{0}$ the inversion formula for the wavelet transform gives

$$
v=C_{u} \int_{\mathbb{R}} \int_{\mathbb{R}} V_{u}(v)(a, b) \pi(a, b) u \frac{d a d b}{a^{2}}
$$

for some constant $C_{u}$ depending only on $u$. Since all non-zero functions in $\mathcal{S}_{0}$ satisfy (5) they are all cyclic in $S_{0}$. We see that $L^{2}(\mathbb{R})$ is weakly dense in $\mathcal{S}_{0}^{\prime}$, and thus $\mathcal{S}_{0}$, which is dense in $L^{2}(\mathbb{R})$ by irreducibility, is also weakly dense in $\mathcal{S}_{0}^{\prime}$.

To check the remaining properties needed for construction of coorbit spaces, let us note the following result which is equivalent to [12, Theorem 19.0.1]

Lemma 4.1. Let

$$
\mathcal{S}\left(\mathbb{R}_{0} \times \mathbb{R}\right)=\left\{v \in \mathcal{S}\left(\mathbb{R}^{2}\right)|\sup |\left(a^{2}+a^{-2}\right)^{n}\left(1+b^{2}\right)^{m} D^{\alpha} \widehat{v}(a, b) \mid<\infty\right\}
$$

then

$$
V_{u}: \mathcal{S}_{0} \rightarrow \mathcal{S}\left(\mathbb{R}_{0} \times \mathbb{R}\right)
$$


is continuous for any $u \in \mathcal{S}_{0}$.

(R1) By Lemma 4.1 we see that $V_{u}(v)$ is in $L^{1}(G)$ and thus also in $L^{2}(G)$ when $u, v \in \mathcal{S}_{0}(\mathbb{R})$. Therefore we can pick a $u \in \mathcal{S}_{0}$ such that $V_{u}(v) * V_{u}(u)=V_{u}(v)$ for all $v \in \mathcal{S}_{0}$.

(R2) Let $Y$ be the mixed norm Banach space

$$
L_{s}^{p, q}(G)=\left\{f \mid\left(\int_{\mathbb{R}^{*}}\left(\int_{\mathbb{R}}|f(a, b)|^{p} a^{-s} d b\right)^{/ p} \frac{d a}{a^{2}}\right)^{1 / q}<\infty\right\}
$$

for $p, q \geq 1$ and $s \in \mathbb{R}$. Then by Minkowski's inequality

$$
\left\|f * V_{u}(u)\right\|_{L_{s}^{p, q}} \leq\|f\|_{L_{s}^{p, q}}\left\|V_{u}(u)\right\|_{L_{s}^{1}}
$$

which shows that $f \mapsto f * V_{u}(u)$ is continuous.

(R3) From Lemma 4.1 it is obtained that for $1 \leq p^{\prime}, q^{\prime} \leq \infty$ the voice transform $V_{u}(u)$ is in $L_{-s}^{p^{\prime}, q^{\prime}}(G)$. Then Hölders inequality tells us that $f * V_{u}(u) \in L_{-s}^{\infty}(G)$ if we pick $p^{\prime}$ and $q^{\prime}$ such that $1 / p+1 / p^{\prime}=1$ and $1 / q+1 / q^{\prime}=1$. So if $f=f * V_{u}(u)$ it will follow that $f \in L_{-s}^{\infty}(G)$ and then

$$
\mathcal{S}_{0} \ni v \mapsto \int_{\mathbb{R}} \int_{\mathbb{R}} f(a, b)\langle v, \pi(a, b) u\rangle \frac{d a d b}{a^{2}}
$$

is continuous, since the mapping $S_{0} \ni v \mapsto V_{u}(v) \in L_{s}^{1}(G)$ is continuous.

(R4) That

$$
\mathcal{S}_{0}^{\prime} \ni v^{\prime} \mapsto \int_{\mathbb{R}} \int_{\mathbb{R}}\left\langle v^{\prime}, \pi(a, b) u\right\rangle\langle\pi(a, b) u, u\rangle \frac{d a d b}{a^{2}}
$$

is weakly continuous is exactly the statement of Theorem 24.1.1 in [12].

This verifies that the space

$$
\mathrm{Co}_{\mathcal{S}_{0}}^{u} L_{s}^{p, q}(G)=\left\{v^{\prime} \in \mathcal{S}_{0}^{\prime} \mid V_{u}(v) \in L_{s}^{p, q}(G)\right\}
$$

is Banach a space. Since the representation $\pi$ is integrable this space coincides with the coorbit space $\mathrm{Co}_{F G} L_{s}^{p, q}(G)$ of Feichtinger and Gröchenig. In [4, 9] it is proven that the homogeneous Besov spaces (see [20])

$$
\dot{B}_{p, q}^{s}=\left\{f \in S_{0}^{\prime} \mid \sum_{j \in \mathbb{Z}} 2^{s j q}\left\|\mathcal{F}^{-1}\left(\phi_{j} \mathcal{F} f\right)\right\|_{L^{p}}^{q}<\infty\right\}
$$

can be characterized as $\operatorname{Co}_{\mathcal{S}_{0}}^{u} L_{s+1 / 2-1 / q}^{p, q}(G)$. Here $\phi \in \mathcal{S}_{0}$ and $\phi_{j}(x)=\phi\left(2^{-j} x\right)$ are chosen such that $\operatorname{supp}(\widehat{\phi}) \subseteq\{x|1 / 2 \leq| x \mid \leq 2\}$ and $\sum_{j \in \mathbb{Z}} \phi_{j}=1$.

4.3. Coorbit spaces for Sobolev spaces on the $(\boldsymbol{a x}+\boldsymbol{b})$-group. We now present a type of coorbit spaces which cannot be described by previous coorbit theories. In particular we find coorbit spaces related to the non-solid Sobolev spaces on the $(a x+b)$-group.

Again let $G$ be the $(a x+b)$-group with representation $(\pi, \mathcal{H})$ as before. The $(a x+b)$-group can also be regarded as a matrix group

$$
G \simeq\left\{\left(\begin{array}{ll}
a & b \\
0 & 1
\end{array}\right) \mid a \neq 0, b \in \mathbb{R}\right\}
$$

The matrices

$$
X_{1}=\left(\begin{array}{ll}
1 & 0 \\
0 & 0
\end{array}\right) \quad \text { and } \quad X_{2}=\left(\begin{array}{cc}
0 & 1 \\
0 & 0
\end{array}\right)
$$


form a basis for the Lie algebra $\mathfrak{g}$. The representation $\pi$ induces the following differential operators

$$
\pi\left(X_{1}\right) f(x)=-\frac{1}{2} f(x)-x f^{\prime}(x) \quad \text { and } \quad \pi\left(X_{2}\right) f(x)=-f^{\prime}(x) .
$$

For $f \in C^{\infty}(G)$ and $X \in \mathfrak{g}$ let

$$
X f(x)=\left.\frac{d}{d t}\right|_{t=0} f(\exp (-t X) x)
$$

For $p \geq 1$ define the Sobolev space (see [19, Definition 3])

$$
W_{p}^{m}(G)=\left\{f \in L^{p}(G) \mid\|f\|_{W_{p}^{m}}=\sum_{k=1}^{m} \sum_{n_{k} \in\{1,2\}}\left\|X_{n_{1}} \cdots X_{n_{k}} f\right\|_{L^{p}}<\infty\right\}
$$

As in the previous section we use the Gelfand triple $\left(\mathcal{S}_{0}, \mathcal{H}, \mathcal{S}_{0}^{*}\right)$ for which the conditions (R1) and (4) are satisfied. Since $W_{p}^{m}(G) \subseteq L^{p}(G)$ and $V_{u}(v) \in\left(L^{p}(G)\right)^{*}$ for $p \geq 1$ the condition (R3) is also satisfied. It remains to show that

$$
W_{p}^{m}(G) \ni f \mapsto f * V_{u}(u) \in W_{p}^{m}(G)
$$

is a continuous mapping for some $u \in \mathcal{S}_{0}$. We note again that by Lemma $4.1 V_{u}(v) \in L^{1}(G)$ for any $u, v \in \mathcal{S}_{0}$ and therefore $f * V_{u}(u) \in L^{p}(G)$ for $f \in W_{p}^{m}(G)$. Finally

$$
X_{i}\left(f * V_{u}(u)\right)=f * V_{\pi\left(X_{i}\right) u}(u)
$$

and since $\pi\left(X_{i}\right) \mathcal{S}_{0} \subseteq \mathcal{S}_{0}$ it follows that $X_{i}\left(f * V_{u}(u)\right) \in L^{p}(G)$. Thus $f * V_{u}(u) \in W_{p}^{m}(G)$ if $f \in L^{p}(G)$, and $W_{p}^{m}(G) \ni f \mapsto f * V_{u}(u) \in W_{p}^{m}(G)$ is continuous. Therefore the space

$$
\mathrm{Co}_{\mathcal{S}_{0}}^{u} W_{p}^{m}(G)=\left\{v^{\prime} \in \mathcal{S}_{0} \mid V_{u}\left(v^{\prime}\right) \in W_{p}^{m}(G)\right\}
$$

is a Banach space. Notice that $W_{p}^{m}(G)$ is not solid for $m \geq 1$ (the characteristic functions for compact intervals are not in $\left.W_{p}^{m}(G)\right)$.

4.4. Discrete series representation for $\mathrm{SU}(\mathbf{1}, \mathbf{1})$ and the $(\boldsymbol{a x}+\boldsymbol{b})$-group. In this section we show that some Bergman spaces can be described as coorbit spaces related to the discrete series representations $\pi_{n}$ of $\mathrm{SL}_{2}(\mathbb{R})$ for integers $n \geq 2$. The construction will also work for representations $\pi_{s}$ for non-integer parameters $s>1$ if we replace $S L_{2}(\mathbb{R})$ by a covering group. This has been done before in [4] in the case of the integrable representations. In that paper it is also noted, that it should be possible to define coorbits for a non-integrable discrete series representation. With the generalized coorbit space theory, this is indeed possible as we will show in the following. Furthermore we also show that the discretization procedures of [5, 9] carry over to this non-integrable case.

The example can be generalized to other bounded symmetric domains, but that would require too much new notation to carry out here.

4.4.1. Continuous description. The connected $(a x+b)$-group $G$ can be realized as the group

$$
G=\left\{\left(\begin{array}{cc}
a & b \\
0 & a^{-1}
\end{array}\right) \mid a>0, b \in \mathbb{R}\right\} \subseteq \mathrm{SL}_{2}(\mathbb{R})
$$


which can be regarded as a subgroup of $\mathrm{SU}(1,1)$ using the Cayley transform:

$$
\begin{aligned}
\left(\begin{array}{cc}
\alpha & \beta \\
\bar{\beta} & \bar{\alpha}
\end{array}\right) & =\frac{1}{2}\left(\begin{array}{cc}
1 & -i \\
-i & 1
\end{array}\right)\left(\begin{array}{cc}
a & b \\
0 & a^{-1}
\end{array}\right)\left(\begin{array}{cc}
1 & i \\
i & 1
\end{array}\right) \\
& =\frac{1}{2}\left(\begin{array}{cc}
a+a^{-1}+i b & b+i\left(a-a^{-1}\right) \\
b-i\left(a-a^{-1}\right) & a+a^{-1}-i b
\end{array}\right)
\end{aligned}
$$

Let $\mathbb{D}$ be the unit disc $\mathbb{D}=\{z \in \mathbb{C}|| z \mid<1\}$ and $n \geq 2$ an integer, then the pairing

$$
(u, v)_{n}=\int_{\mathbb{D}} u(z) \overline{v(z)}\left(1-|z|^{2}\right)^{n-2} d x d y
$$

gives and inner product on the Hilbert space

$$
\mathcal{H}_{n}=\mathcal{A}^{2, n}(\mathbb{D})=\left\{v \in \mathcal{O}(\mathbb{D}) \mid(v, v)_{n}<\infty\right\} .
$$

The discrete series representation

$$
\pi_{n}\left(\begin{array}{cc}
\alpha & \beta \\
\bar{\beta} & \bar{\alpha}
\end{array}\right) v(z)=(-\bar{\beta} z+\bar{\alpha})^{-n} v\left(\frac{\alpha z-\beta}{-\bar{\beta} z+\bar{\alpha}}\right)
$$

is an irreducible unitary representation of $G$ on $\mathcal{H}_{n}=\mathcal{A}^{2, n}(\mathbb{D})($ see $[10,11,13,15])$.

As distribution space $S^{*}$ we will use the conjugate dual of the space $S=\mathcal{H}_{n}^{\infty}$. We will denote the conjugate dual by $\mathcal{H}_{n}^{-\infty}$.

The function $\psi(z)=1$ is in $\mathcal{H}_{n}^{\infty}$ for all $n$. Since $\left(\pi_{n}, \mathcal{H}_{n}^{\infty}\right)$ is irreducible as a representation $S L_{2}(\mathbb{R})$ (see [21, p. 254]), the function $\psi$ is $\mathrm{SL}_{2}(\mathbb{R})$-cyclic in $\mathcal{H}_{n}^{\infty}$. The group $S U(1,1)$ has the Iwasawa decomposition $N A K$ where

$$
\begin{aligned}
& N=\left\{\left(\begin{array}{cc}
1+i r & r \\
r & 1-i r
\end{array}\right) \mid r \in \mathbb{R}\right\}, \\
& A=\left\{\left(\begin{array}{cc}
\cosh s & i \sinh s \\
-i \sinh s & \cosh s
\end{array}\right) \mid s \in \mathbb{R}\right\} \\
& K=\left\{\left(\begin{array}{cc}
e^{i t} & 0 \\
0 & e^{-i t}
\end{array}\right) \mid t \in \mathbb{R}\right\},
\end{aligned}
$$

The action of an element in $K$ on $\psi$ is

$$
\pi_{n}\left(\begin{array}{cc}
e^{i t} & 0 \\
0 & e^{-i t}
\end{array}\right) \psi(z)=e^{i n t} \psi(z),
$$

and hence $\psi$ is also $N A$-cyclic in $\mathcal{H}_{n}^{\infty}$. The group $N A$ is exactly the subgroup of $\operatorname{SU}(1,1)$ corresponding to $G$, so we conclude that $\psi$ is $G$-cyclic in $\mathcal{H}_{n}^{\infty}$.

By use of polar coordinates and Cauchy's integral theorem the voice transform $V_{\psi}(\psi)$ is

$$
V_{\psi}(\psi)\left(\begin{array}{cc}
\alpha & \beta \\
\bar{\beta} & \bar{\alpha}
\end{array}\right)=\int_{\mathbb{D}}(-\bar{\beta} z+\bar{\alpha})^{-n}\left(1-|z|^{2}\right)^{n-2} d x d y=\frac{1}{2(n-1) \bar{\alpha}^{n}}
$$

As a function of $(a, b)$ we have

$$
F_{n}(a, b)=V_{\psi}(\psi)\left(\begin{array}{cc}
\alpha & \beta \\
\bar{\beta} & \bar{\alpha}
\end{array}\right)=\frac{1}{2(n-1)}\left(a+a^{-1}-i b\right)^{-n} e^{-i n t} .
$$

Furthermore

$$
\left\|F_{n}\right\|_{L^{p}(G)}^{p}=\frac{1}{2^{p}(n-1)^{p}} \int_{-\infty}^{\infty} \int_{0}^{\infty} \frac{1}{\left(\left(a+a^{-1}\right)^{2}+b^{2}\right)^{n p / 2}} \frac{d a d b}{a^{2}}<\infty
$$


if and only if $n p>2$. In particular we recover the well know fact that $F_{n}$ is square integrable when $n \geq 2$ and integrable for $n \geq 3$.

While $F_{2}$ is not in $L^{1}(G)$ it is very close in the following sense

Lemma 4.2. If $0<\epsilon<2$ then

$$
\int_{0}^{\infty} \int_{-\infty}^{\infty}\left|F_{2}(a, b)\right| a^{\epsilon} \frac{d b d a}{a^{2}}<\infty
$$

This can be used to show that the conditions for construction of coorbit spaces are satisfied.

(R1) is satisfied since $F_{n} \in L^{2}(G)$ for $n \geq 2$, showing that the representations $\pi_{n}$ are square integrable. Let $u$ be a normalization of $\psi$ in order to make convolution with $V_{u}(u)$ an idempotent.

(R2) The mapping $f \mapsto f * F_{n}$ is bounded from $L^{p}(G)$ to $L^{p}(G)$ for $p>1$. Using Minkowski's inequality it is easy to see for $n \geq 3$, since then $F_{n}$ is in $L^{1}(G)$. We will show that it is also true for $n=2$. Assume that $f \in L^{p}(G)$ and pick $q$ such that $1 / p+1 / q=1$, and look at

$$
\begin{aligned}
&\left|\iint f(a, b) F_{2}\left((a, b)^{-1}\left(a_{1}, b_{1}\right)\right) \frac{d a d b}{a^{2}}\right|^{p} \\
& \leq\left(\iint|f(a, b)| a^{-1 / p q}\left|F_{2}\left((a, b)^{-1}\left(a_{1}, b_{1}\right)\right)\right|^{1 / p+1 / q} a^{1 / p q} \frac{d a d b}{a^{2}}\right)^{p} \\
& \leq\left(\iint|f(a, b)|^{p} a^{-1 / q}\left|F_{2}\left((a, b)^{-1}\left(a_{1}, b_{1}\right)\right)\right| \frac{d a d b}{a^{2}}\right) \\
& \times\left(\iint a^{1 / p}\left|F_{2}\left((a, b)^{-1}\left(a_{1}, b_{1}\right)\right)\right| \frac{d a d b}{a^{2}}\right)^{p / q}
\end{aligned}
$$

By the unitarity of $\pi_{n}$ it is true that $\left|F_{n}((a, b))\right|=\left|F_{n}\left((a, b)^{-1}\right)\right|$ so the second integral becomes

$$
\iint a^{1 / p}\left|F_{2}\left(\left(a_{1}, b_{1}\right)^{-1}(a, b)\right)\right| \frac{d a d b}{a^{2}}=\iint\left(a a_{1}\right)^{1 / p}\left|F_{2}((a, b))\right| \frac{d a d b}{a^{2}} \leq C a_{1}^{1 / p}
$$

where the have used the invariance of the measure and Lemma 4.2. Thus we get

$$
\begin{aligned}
& \left|\iint f(a, b) F_{2}\left((a, b)^{-1}\left(a_{1}, b_{1}\right)\right) \frac{d a d b}{a^{2}}\right|^{p} \\
& \quad \leq C \iint|f(a, b)|^{p} a^{-1 / q}\left|F_{2}\left((a, b)^{-1}\left(a_{1}, b_{1}\right)\right)\right| \frac{d a d b}{a^{2}} a_{1}^{1 / q} .
\end{aligned}
$$

Now we can calculate an estimate for the norm using Fubuni's theorem and (6) again

$$
\begin{aligned}
\left\|f * F_{2}\right\|_{p}^{p} & \leq C \iiint \int|f(a, b)|^{p} a^{-1 / q}\left|F_{2}\left((a, b)^{-1}\left(a_{1}, b_{1}\right)\right)\right| \frac{d a d b}{a^{2}} a_{1}^{1 / q} \frac{d a_{1} d b_{1}}{a_{1}^{2}} \\
& =C \iint|f(a, b)|^{p} a^{-1 / q} \iint\left|F_{2}\left((a, b)^{-1}\left(a_{1}, b_{1}\right)\right)\right| a_{1}^{1 / q} \frac{d a_{1} d b_{1}}{a_{1}^{2}} \frac{d a d b}{a^{2}} \\
& \leq C^{2} \iint|f(a, b)|^{p} a^{-1 / q} a^{1 / q} \frac{d a d b}{a^{2}} \\
& =C^{2}\|f\|_{p}^{p}
\end{aligned}
$$

This shows that $f \mapsto f * F_{n}$ is a continuous linear operator on $L^{p}(G)$ for all $n \geq 2$. 
(R3) The smooth vectors for the representation $\pi_{n}$ have been characterized in [15] and more generally in [1] to be

$$
\mathcal{H}_{n}^{\infty}=\left\{\left.\sum_{k=0}^{\infty} a_{k} z^{k}\left|\forall m: \sum_{k=0}^{\infty}\right| a_{k}\right|^{2} \frac{(n-1) ! k !}{(n+k-1) !}\left(n(n-2)+2 k^{2}\right)^{2 m}<\infty\right\}
$$

with conjugate dual consisting of formal power series

$$
\mathcal{H}_{n}^{-\infty}=\left\{\left.\sum_{k=0}^{\infty} b_{k} z^{k}\left|\exists m: \sum_{k=1}^{\infty}\right| b_{k}\right|^{2} \frac{(n-1) ! k !}{(n+k-1) !}\left(n(n-2)+2 k^{2}\right)^{-2 m}<\infty\right\}
$$

Let $\phi$ be a smooth vector with expansion $\sum_{k=0}^{\infty} a_{k} z^{k}$. The Taylor series for $\pi\left(\begin{array}{cc}\alpha & \beta \\ \bar{\beta} & \bar{\alpha}\end{array}\right) 1(z)$ is

$$
\pi\left(\begin{array}{cc}
\alpha & \beta \\
\bar{\beta} & \bar{\alpha}
\end{array}\right) 1(z)=\alpha^{-n} \sum_{k=0}^{\infty}(\beta / \alpha)^{k}(-1)^{k} \frac{(n+k-1) !}{(n-1) ! k !} z^{k}
$$

Therefore, since $\left(z^{k}, z^{k}\right)_{n}=k !(n-2) ! /(k+n-1) !$,

$$
V_{\psi}(\phi)(a, b)=\frac{1}{(n-1) \bar{\alpha}^{n}} \sum_{k=0}^{\infty}(-1)^{k}(\beta / \alpha)^{k} a_{k}=2 F_{n}(a, b) \sum_{k=0}^{\infty}(-1)^{k}(\beta / \alpha)^{k} a_{k}
$$

and it can estimated by

$$
\left|V_{1}(\phi)(a, b)\right| \leq 2\left|F_{n}(a, b)\right| \sum_{k=0}^{\infty}\left|a_{k}\right|
$$

since $|\alpha|>|\beta|$. For any $m$ we obtain that

$$
\begin{aligned}
\sum_{k=0}^{\infty}\left|a_{k}\right| \leq\left|a_{0}\right| & +\left(\sum_{k=1}^{\infty} \frac{(n+k-1) !}{k !(n-1) !}\left(n(n-2)+2 k^{2}\right)^{-2 m}\right)^{1 / 2} \\
& \times\left(\sum_{k=1}^{\infty}\left|a_{k}\right|^{2} \frac{(n-1) ! k !}{(n+k-1) !}\left(n(n-2)+2 k^{2}\right)^{2 m}\right)^{1 / 2}
\end{aligned}
$$

and by picking $m$ large enough the first sum converges. Thus the mapping $\phi \mapsto V_{\psi}(\phi)$ is continuous from $\mathcal{H}_{\pi}^{\infty}$ to $L^{q}$ for all $q>1$. So if $f \in L^{p}(G)$ for $p>1$ then the mapping $\phi \mapsto \int f(a, b) V_{\psi}(\phi)(a, b) \frac{d a d b}{a^{2}}$ is continuous by Hölder's inequality. This ensures the validity of (주).

(4) By Proposition 3.2 the condition ( $(\underline{\mathrm{R}} 4)$ is automatically satisfied when $u$ is a constant multiple of $\psi \in \mathcal{H}_{n}^{\infty}$.

This means that all the conditions in Assumption 2.1 are satisfied for some $u \in \mathcal{H}_{n}^{\infty}$ (constant multiple of $\psi(z)=1$ ) with the Banach space $Y=L^{p}(G)$. Therefore the space

$$
\mathrm{Co}_{\mathcal{H}_{n}^{\infty}}^{u} L^{p}(G)=\left\{v^{\prime} \in \mathcal{H}_{n}^{-\infty} \mid V_{u}\left(v^{\prime}\right) \in L^{p}(G)\right\}
$$

is a Banach space. As pointed out in [4, Section 7] these spaces are the Bergman spaces $\mathcal{A}^{p, p n / 2}$ where

$$
\mathcal{A}^{p, \alpha}(\mathbb{D})=\left\{f \in \mathcal{O}\left(\left.\mathbb{D}\left|\int_{\mathbb{D}}\right| f(z)\right|^{p}\left(1-|z|^{2}\right)^{\alpha-2} d z<\infty\right\}\right.
$$


4.4.2. Discretization. In the following we will only work with the non-integrable voice transform $F_{2}=V_{\psi}(\psi)$ since the other cases are covered by the theory of Feichtinger and Gröchenig. The aim is to show that discretizations of coorbit spaces are possible without integrable representations.

The key to finding atomic decompositions will be the following result

Lemma 4.3. For each $\epsilon>0$ there is a neighbourhood $U$ of the identity such that

$$
\left|\frac{F_{2}((a, b)(x, y))}{F_{2}(x, y)}-1\right|<\epsilon
$$

for $(a, b) \in U$.

Proof.

$$
\begin{aligned}
\left|\frac{F_{2}((a, b)(x, y))}{F_{2}(x, y)}-1\right|^{2}= & \left|\frac{\left(a x+(a x)^{-1}-i\left(b x^{-1}+a y\right)\right)^{2}-\left(x+x^{-1}-i y\right)^{2}}{\left(x+x^{-1}-i y\right)^{2}}\right|^{2} \\
= & \left|\frac{a x+(a x)^{-1}-i\left(b x^{-1}+a y\right)+\left(x+x^{-1}-i y\right)^{2}}{x+x^{-1}-i y}\right|^{2} \\
= & \times\left|\frac{(1+a) x+\left(1+a^{-1} x^{-1}-i\left(b x^{-1}+(1+a) y\right.\right.}{x+x^{-1}-i y}\right|^{2} \\
& \times\left|\frac{(a-1) x+\left(a^{-1}-1\right) x^{-1}-i\left(b x^{-1}+(a-1) y\right)}{x+x^{-1}-i y}\right|^{2}
\end{aligned}
$$

Let $1 / \delta<a<\delta$ for $\delta>1$ and $-\gamma<b<\gamma$ for $\gamma>0$ and then estimate each of the squares

$$
\begin{aligned}
& \left|\frac{(1+a) x+\left(1+a^{-1} x^{-1}-i\left(b x^{-1}+(1+a) y\right.\right.}{x+x^{-1}-i y}\right|^{2} \\
& \quad=\frac{\left((1+a) x+\left(1+a^{-1} x^{-1}\right)^{2}+\left(\left(b x^{-1}+(1+a) y\right)^{2}\right.\right.}{\left(x+x^{-1}\right)^{2}+y^{2}} \\
& \quad \leq \frac{\left((1+\delta) x+(1+\delta) x^{-1}\right)^{2}}{\left(x+x^{-1}\right)^{2}+y^{2}}+\frac{b^{2} x^{-2}}{\left(x+x^{-1}\right)^{2}+y^{2}}+\frac{(1+\delta)^{2} y^{2}}{\left(x+x^{-1}\right)^{2}+y^{2}}+\frac{2(1+\delta)|b||y| x^{-1}}{\left(x+x^{-1}\right)^{2}+y^{2}} \\
& \quad \leq(1+\delta)^{2}+b^{2}+(1+\delta)^{2}+2(1+\delta)|b| \frac{x^{-1}}{\sqrt{\left(x+x^{-1}\right)^{2}+y^{2}}} \frac{x^{\left(x+x^{-1}\right)^{2}+y^{2}}}{\sqrt{(1+\delta)^{2}+\gamma^{2}+(1+\delta)^{2}+2(1+\delta) \gamma}} \\
& \quad \leq(1)
\end{aligned}
$$


This we can get arbitrarily close to 8 by picking $\delta$ and $\gamma$ close enough to 1 and 0 respectively. Similarily

$$
\begin{aligned}
& \left|\frac{(a-1) x+\left(a^{-1}-1\right) x^{-1}-i\left(b x^{-1}+(a-1) y\right.}{x+x^{-1}-i y}\right|^{2} \\
& \quad=\frac{\left((a-1) x+\left(a^{-1}-1\right) x^{-1}\right)^{2}+\left(\left(b x^{-1}+(a-1) y\right)^{2}\right.}{\left(x+x^{-1}\right)^{2}+y^{2}} \\
& \quad \leq \frac{\left((\delta-1) x+(\delta-1) x^{-1}\right)^{2}}{\left(x+x^{-1}\right)^{2}+y^{2}}+\frac{b^{2} x^{-2}}{\left(x+x^{-1}\right)^{2}+y^{2}}+\frac{(a-1)^{2} y^{2}}{\left(x+x^{-1}\right)^{2}+y^{2}}+\frac{2(\delta-1)|b||y| x^{-1}}{\left(x+x^{-1}\right)^{2}+y^{2}} \\
& \quad \leq(\delta-1)^{2}+\gamma^{2}+(\delta-1)^{2}+2(\delta-1) \gamma
\end{aligned}
$$

This last term can be made arbitrarily close to 0, thus finishing the proof of the lemma.

From this result follows easily

Corollary 4.4. There exist a neighbourhood $U$ of the identity and constants $C_{1}, C_{2}>0$ such that

$$
C_{1}\left|F_{2}(x, y)\right| \leq\left|F_{2}((a, b)(x, y))\right| \leq C_{2}\left|F_{2}(x, y)\right|
$$

for all $(x, y) \in G$ with $(a, b) \in U$. These constants can be chosen arbitrarily close to 1 , by choosing $U$ small enough.

When discretizing the reproducing formula, we need the following definition from [5]:

Definition 4.5. Let $V$ be a compact neighbourhood of the identity. The set of points $\left\{x_{i}\right\}$ are said to be $V$-separated if the $x_{i} V$ are pairwise disjoint.

Let $U$ be a compact neighbourhood of the identity. The set of points $\left\{x_{i}\right\}$ are said to be $U$-dense if $G=\cup_{i} x_{i} U$.

Proposition 4.6. Let $V \subseteq U$ be compact neighbourhoods of the identitiy. Assume that the points $\left\{x_{i}\right\}$ are $V$-separated and $U$-dense and that $U$ satisfies Corollary 4.4, Let $\left\{\psi_{i}\right\}$ be a partition of unity for which $\operatorname{supp}\left(\psi_{i}\right) \subseteq x_{i} U$. Then the following is true

(a) The mapping $\ell^{p} \ni\left(\lambda_{i}\right) \mapsto \sum_{i} \lambda_{i} \ell_{x_{i}} F_{2} \in L^{p}(G) * F_{2}$ is continuous

(b) The mapping $L^{p}(G) * F_{2} \ni f \mapsto\left(f\left(x_{i}\right)\right)_{i \in I} \in \ell^{p}(I)$ is continuous

(c) The mapping $L^{p}(G) * F_{2} \ni f \mapsto\left(\int_{G} f(x) \psi_{i}(x) d x\right)_{i \in I} \in \ell^{p}(I)$ is continuous

Proof. First note that the norms on $\ell^{p}$ and $L^{p}(G)$ are related in the following sense

$$
\left\|\left(\lambda_{i}\right)\right\|_{\ell^{p}}=\frac{1}{|V|}\left\|\sum_{i} \lambda_{i} 1_{x_{i} V}\right\|_{L^{p}}
$$

Also the convolution in $L^{p}(G)$ with $\left|F_{2}\right|$ is continuous and we will denote the norm of this convolution by $D_{p}$. (国) If $\left(\lambda_{i}\right) \in \ell^{p}$ then the function

$$
f=\sum_{i}\left|\lambda_{i}\right| 1_{x_{i} V}
$$

is in $L^{p}(G)$ and $\|f\|_{L^{p}(G)}=|V|\left\|\left(\lambda_{i}\right)\right\|_{\ell^{p}}$. Convolution with $\left|F_{2}\right|$ is continuous so

$$
f *\left|F_{2}\right|=\sum_{i}\left|\lambda_{i}\right| 1_{x_{i} V} *\left|F_{2}\right|
$$


is in $L^{p}(G)$. Now let us show that the function $1_{x_{i} V} *\left|F_{2}\right|$ is bigger than some constant times $\ell_{x_{i}}\left|F_{2}\right|$.

$$
\int 1_{x_{i} V}(z)\left|F_{2}\left(z^{-1} y\right)\right| d z=\int_{V}\left|F_{2}\left(z^{-1} x_{i}^{-1} y\right)\right| d z \geq C_{1}|V|\left|F_{2}\left(x_{i}^{-1} y\right)\right|
$$

This shows that

$$
\begin{aligned}
\left|\sum_{i} \lambda_{i} \ell_{x_{i}} F_{2}(y)\right| & \leq \sum_{i}\left|\lambda_{i}\right|\left|F_{2}\left(x_{i}^{-1} y\right)\right| \\
& \leq \frac{1}{C_{1}|V|} \sum_{i}\left|\lambda_{i}\right| 1_{x_{i} V} *\left|F_{2}\right|(y) \\
& =\frac{1}{C_{1}|V|} f *\left|F_{2}\right| .
\end{aligned}
$$

Since $f *\left|F_{2}\right| \in L^{p}(G)$ the sum $\sum_{i} \lambda_{i} \ell_{x_{i}} F_{2}$ is in $L^{p}(G)$ with norm

$$
\begin{aligned}
\left\|\sum_{i} \lambda_{i} \ell_{x_{i}} F_{2}(y)\right\|_{L^{p}} & \leq \frac{1}{C_{1}|V|}\left\|f *\left|F_{2}\right|\right\|_{L^{p}} \\
& \leq \frac{D_{p}}{C_{1}|V|}\|f\|_{L^{p}} \\
& =\frac{D_{p}}{C_{1}}\left\|\left(\lambda_{i}\right)\right\|_{\ell^{p}} .
\end{aligned}
$$

This shows the desired continuity.

(b) We need to show that $f\left(x_{i}\right)$ is in $\ell^{p}$, but this is the same as showing that $g=$ $\sum_{i}\left|f\left(x_{i}\right)\right| 1_{x_{i} V}$ is in $L^{p}(G) . f \in L^{p}(G) * F_{2}$ so

$$
\sum_{i}\left|f\left(x_{i}\right)\right| 1_{x_{i} V}(y) \leq \sum_{i}|f| *\left|F_{2}\right|\left(x_{i}\right) 1_{x_{i} V}(y)=\int|f(z)| \sum_{i}\left|F_{2}\left(z^{-1} x_{i}\right)\right| 1_{x_{i} V}(y) d z
$$

For each $y$ at most one $i$ adds to this sum, namely the $i$ for which $x_{i} \in y V^{-1}$. Therefore

$$
\sum_{i}\left|F_{2}\left(z^{-1} x_{i}\right)\right| 1_{x_{i} V}(y) \leq \sup _{v \in V}\left|F_{2}\left(z^{-1} y v^{-1}\right)\right| \leq C_{2}\left|F_{2}\left(z^{-1} y\right)\right|
$$

by Corollary 4.4. We then get

$$
\sum_{i}\left|f\left(x_{i}\right)\right| 1_{x_{i} V}(y) \leq C_{2} \int|f(z)|\left|F_{2}\left(z^{-1} y\right)\right| d z=C_{2}|f| *\left|F_{2}\right|(y)
$$

and finally

$$
\left\|f\left(x_{i}\right)\right\|_{\ell^{p}} \leq \frac{C_{2} D_{p}}{|V|}\|f\|_{L^{p}}
$$

(c) We have to show that the function

$$
\sum_{i}\left(\int f(x) \psi_{i}(x) d x\right) 1_{x_{i} V} \in L^{p}(G)
$$

We get that

$$
\left|\sum_{i}\left(\int f(x) \psi_{i}(x) d x\right) 1_{x_{i} V}(y)\right| \leq \int|f(x)| \sum_{i \in I} \psi_{i}(x) 1_{x_{i} V}(y) d x
$$


and since

$$
\sum_{i} \psi_{i}(x) 1_{x_{i} V}(y) \leq \sum_{i} 1_{x_{i} U}(x) 1_{x_{i} V}(y) \leq 1_{U^{-1} V}\left(x^{-1} y\right)
$$

we obtain

$$
\left|\sum_{i}\left(\int f(x) \psi_{i}(x) d x\right) 1_{x_{i} V}(y)\right| \leq \int|f(x)| 1_{U^{-1} V}\left(x^{-1} y\right) d x=|f| * 1_{U^{-1} V}(y)
$$

which is in $L^{p}(G)$ with norm continuously dependent on $f$, i.e.

$$
\left\|\sum_{i}\left(\int f(x) \psi_{i}(x) d x\right) 1_{x_{i} V}\right\| \leq C\|f\|_{L^{p}}
$$

for some $C>0$.

Proposition 4.7. We can choose a compact neighbourhood $U, U$-dense points $\left\{x_{i}\right\}$ and a partition $\psi_{i}$ of unity with $\operatorname{supp}\left(\psi_{i}\right) \subseteq x_{i} U$ such that the operators defined below are invertible with continuous inverses

(a) define $S: L^{p}(G) * F_{2} \rightarrow L^{p}(G) * F_{2}$ by

$$
S f=\sum_{i} f\left(x_{i}\right) \psi_{i} * F_{2}
$$

(b) define $T: L^{p}(G) * F_{2} \rightarrow L^{p}(G) * F_{2}$ by (with $c_{i}=\int \psi_{i}$ )

$$
T f=\sum_{i} c_{i} f\left(x_{i}\right) \ell_{x_{i}} F_{2}
$$

(c) define $R: L^{p}(G) * F_{2} \rightarrow L^{p}(G) * F_{2}$ by

$$
R f=\sum_{i}\left(\int f(x) \psi_{i}(x) d x\right) \ell_{x_{i}} F_{2}
$$

Proof. For each neighbourhood of the identity $U$ we can pick $U$-dense points $\left\{x_{i}\right\}$ such that $\left\{x_{i}\right\}$ are $V$-separated for some compact neighbourhood of the identity $V$ satisfying $V^{2} \subseteq U$ (see [18, Thm 4.2.2]). Thus we can pick $U$ in order to satisfy the inequality in Lemma 4.3 for any $\epsilon$.

Denote by $D_{p}$ the $L^{p}$ operator norm of convolution by $F_{2}$.

(a) Let $f \in L^{p}(G) * F_{2}$ and let us look at the difference

$$
f(x)-\sum_{i} f\left(x_{i}\right) \psi_{i}(x)=\sum_{i}\left(f(x)-f\left(x_{i}\right)\right) \psi_{i}
$$

For $x \in \operatorname{supp}\left(\psi_{i}\right) \subseteq x_{i} U$ we get

$$
\begin{aligned}
\left|f(x)-f\left(x_{i}\right)\right| & \leq \int|f(z)|\left|F_{2}\left(z^{-1} x\right)-F_{2}\left(z^{-1} x_{i}\right)\right| d z \\
& =\int|f(z)|\left|\frac{F_{2}\left(z^{-1} x_{i}\right)}{F_{2}\left(z^{-1} x\right)}-1\right|\left|F_{2}\left(z^{-1} x\right)\right| d z \quad \leq \epsilon \int|f(z)|\left|F_{2}\left(z^{-1} x\right)\right| d z \\
& =\epsilon|f| *\left|F_{2}\right|(x)
\end{aligned}
$$


This means that

$$
\left|f(x)-\sum_{i} f\left(x_{i}\right) \psi_{i}(x)\right| \leq \epsilon \sum_{i}|f| *\left|F_{2}\right|(x) \psi_{i}(x)=\epsilon|f| *\left|F_{2}\right|(x)
$$

This function is in $L^{p}(G)$ and so

$$
\left\|f-\sum_{i} f\left(x_{i}\right) \psi_{i}\right\|_{L^{p}} \leq \epsilon D_{p}\|f\|_{L^{p}}
$$

Convoluting this expression by $F_{2}$ we get

$$
\left\|f-\sum_{i} f\left(x_{i}\right) \psi_{i} * F_{2}\right\|_{L^{p}} \leq \epsilon D_{p}^{2}\|f\|_{L^{p}}
$$

So picking $U$ such that $\epsilon<D_{p}^{-2}$ we obtain an operator $S$ such that $\|I-S\|<1$ as an operator on $L^{p}(G) * F_{2}$. Therefore $S$ is invertible.

(b) We will show that $T$ is invertible using its difference from the operator $S$.

$$
\begin{aligned}
|T f(x)-S f(x)| & =\left|\sum_{i} f\left(x_{i}\right)\left(\psi_{i} * F_{2}(x)-c_{i} F_{2}\left(x_{i}^{-1} x\right)\right)\right| \\
& \leq \sum_{i}\left|f\left(x_{i}\right)\right|\left|\psi_{i} * F_{2}(x)-c_{i} F_{2}\left(x_{i}^{-1} x\right)\right|
\end{aligned}
$$

Look at

$$
\begin{aligned}
\left|\psi_{i} * F_{2}(x)-c_{i} F_{2}\left(x_{i}^{-1} x\right)\right| & =\mid \int \psi_{i}(z)\left(F_{2}\left(z^{-1} x\right)-F_{2}\left(x_{i}^{-1} x\right) d z \mid\right. \\
& \leq \int \psi_{i}(z)\left|F_{2}\left(z^{-1} x\right)-F_{2}\left(x_{i}^{-1} x\right)\right| d z \\
& \leq \epsilon \int \psi_{i}(z)\left|F_{2}\left(z^{-1} x\right)\right| d z \\
& =\epsilon \psi_{i} *\left|F_{2}\right|(x)
\end{aligned}
$$

Then we have

$$
|T f(x)-S f(x)| \leq \epsilon \sum_{i}\left|f\left(x_{i}\right)\right| \psi_{i} *\left|F_{2}\right|(x)
$$

This is a function in $L^{p}(G)$ and the norm is

$$
\|T f-S f\|_{L^{p}} \leq \epsilon D_{p}\left\|\sum_{i}\left|f\left(x_{i}\right)\right| \psi_{i}\right\|_{L^{p}} \leq \epsilon^{2} D_{p}^{2}\|f\|_{L^{p}}
$$

This means that $\|I-T\| \leq\|I-S\|+\|S-T\| \leq \epsilon D_{p}\left(1+\epsilon D_{p}\right)$ and if we pick $U$ such that this norm is less than 1 we get that $T$ is invertible.

(C) Can be proven by estimates as above.

This now means that any $f \in L^{p}(G) * F_{2}$ can be reconstructed in the following ways (we only write this up for the operator $T$ )

$$
f=\sum_{i} c_{i} f\left(x_{i}\right) T^{-1}\left(\ell_{x_{i}} F_{2}\right)
$$


or

$$
f=\sum_{i} c_{i}\left(T^{-1} f\right)\left(x_{i}\right) \ell_{x_{i}} F_{2}
$$

The first representation in turn means that a $v^{\prime} \in \mathrm{Co}_{\mathcal{H}_{n}^{\infty}}^{u} L^{p}(G)$ can be reconstructed from the samples of its voice transform, i.e.

$$
v^{\prime}=\sum_{i} c_{i} V_{u}\left(v^{\prime}\right)\left(x_{i}\right) V_{u}^{-1} T^{-1} \ell_{x_{i}} V_{u}(u)
$$

Remark 4.8. In [5] it is concluded that the elements $v_{i}=V_{u}^{-1} T^{-1} \ell_{x_{i}} V_{u}(u)$ are in the space $\mathcal{H}_{m}^{1}$. We cannot obtain such a result, since this space does not exist in general. We however claim, that the $v_{i}$ are in all the coorbit spaces $\operatorname{Co}_{\mathcal{H}_{n}^{\infty}}^{u} L^{p}(G)$ for $p>1$ and so the same vectors can be used in all situations.

4.5. Bandlimited functions. In this section we give a examples of coorbit spaces corresponding to cyclic representations that are far from irreducible. The examples are almost tautological, the representation space is the coorbit space itself. This makes it possible to treat sampling of bandlimited functions using the coorbit theory.

Let $G$ be a locally compact group and let $K \subset G$ be a compact subgroup. We assume that $(G, K)$ is a Gelfand pair, i.e. that the algebra $L^{1}(G / K)^{K}$ of left-invariant functions with convolution is commutative. Thus

$$
\left(\ell, L^{2}(G / K)\right) \simeq \int_{\Lambda}^{\oplus}\left(\pi_{\lambda}, \mathcal{H}_{\lambda}\right) d \mu(\lambda)
$$

with multiplicity one. Here $\ell$ stands for the left regular representation $[\ell(x) f](y)=f\left(x^{-1} y\right)$ and $\Lambda$ is a measurable subset of $\widehat{G}$. Those spaces are sometimes called commutative space [22]. For details in the following arguments we refer to [22] or [16] for the case of Riemannian symmetric spaces of the non-compact type. Because of the multiplicity one assumption it follows that $\operatorname{dim} \mathcal{H}_{\lambda}^{K}=1$ for almost all $\lambda \in \Lambda$. Assume that we can choose a measurable field $\Lambda \ni \lambda \mapsto s_{\lambda} \in \mathcal{H}_{\lambda}^{K}$ such that $\left\|s_{\lambda}\right\|=1$ for almost all $\lambda$. Note that $s_{\lambda}$ is then determined up to a constant $c_{\lambda},\left|c_{\lambda}\right|=1$. The function $x \mapsto \pi_{\lambda}(x) s_{\lambda}$ is right $K$-invariant and can therefore be viewed as a function on $G / K$. From now on we will indentify functions on $G / K$ with right $K$-invariant functions on $G$. For $f \in L^{1}(G / K)$ let

$$
\pi_{\lambda}(f) s_{\lambda}=\int_{G} f(x) \pi_{\lambda}(x) s_{\lambda} d x=\int_{G / K} f(x) \pi_{\lambda}(x) s_{\lambda} d x .
$$

The map $\mathcal{F}: L^{2}(G / K) \rightarrow \int_{\Lambda}^{\oplus} \mathcal{H}_{\lambda} d \mu(\lambda)$ defined by

$$
\mathcal{F}(f)(\lambda)=\widehat{f}(\lambda):=\pi_{\lambda}(f) s_{\lambda}
$$

the vector valued Fourier transform on $G / K$, or simply, as we will not be considering another Fourier transform here, the Fourier transform on $G / K$. First $\mathcal{F}(f)$ is only defined for $f \in$ $L^{1}(G / K) \cap L^{2}(G / K)$ but the following argument shows that $\|f\|=\|\mathcal{F}(f)\|$ and hence $\mathcal{F}$ extends to a unitary isomorphism giving a "concrete" realization of the isomorphism (77).

As $\pi_{\lambda}(\ell(x) f)=\pi_{\lambda}(x) \pi_{\lambda}(f)$ and $\pi_{\lambda}(f * g)=\pi_{\lambda}(f) \pi_{\lambda}(g)\left(x \in G, f, g \in L^{1}(G / K) \cap\right.$ $\left.L^{2}(G / K)\right)$ it follows that:

Lemma 4.9. Let $x \in G$ and $f, g \in L^{1}(G / K) \cap L^{2}(G / K)$. Then $\mathcal{F}(\ell(x) f)(\lambda)=\pi_{\lambda}(x) \widehat{f}(\lambda)$ and $\widehat{f * g}(\lambda)=\pi_{\lambda}(f) \widehat{g}(\lambda)$. 
We also have

$$
\begin{aligned}
\operatorname{Tr}\left(\pi_{\lambda}(f)\right) & =\left(\widehat{f}(\lambda), s_{\lambda}\right)_{\lambda} \\
& =\int_{G / K} f(x)\left(\pi_{\lambda}(x) s_{\lambda}, s_{\lambda}\right)_{\lambda} d x \\
& =\int_{G / K} f(x) \overline{\varphi_{\lambda}(x)} d x
\end{aligned}
$$

where $\varphi_{\lambda}$ is the spherical function $\varphi_{\lambda}(x)=\left(s_{\lambda}, \pi_{\lambda}(x) s_{\lambda}\right)_{\lambda}$. The spherical function is independent of the choice of $s_{\lambda}$. Using the well known theory of the spherical Fourier transform [22] or the abstract Plancherel formula it follows that for $f \in C_{c}(G / K)$ we can choose the measure $\mu$ on $\Lambda$ such that

$$
f(e K)=\int_{\lambda}\left(\widehat{f}(\lambda), s_{\lambda}\right)_{\lambda} d \mu(\lambda) .
$$

Define $f^{*}(x)=\Delta\left(x^{-1}\right) \overline{f\left(x^{-1}\right)}$. Then $\pi_{\lambda}\left(f^{*}\right)=\pi_{\lambda}(f)^{*}$. Thus $\widehat{f^{*} * f}=\pi_{\lambda}(f)^{*} \widehat{f}(\lambda)$ and hence

$$
\|f\|_{2}^{2}=\int_{\Lambda}\|\widehat{f}(\lambda)\|_{\lambda}^{2} d \mu(\lambda)
$$

Lemma 4.10. Let $f \in L^{1}(G / K) \cap L^{2}(G / K)$ be such that $\lambda \mapsto\|\widehat{f}(\lambda)\|$ is integrable. Then $f$ is continuous and

$$
f(x)=\int_{\Lambda} f * \varphi_{\lambda}(x) d \mu(\lambda)=\int_{\Lambda}\left(\widehat{f}(\lambda), \pi_{\lambda}(x) s_{\lambda}\right)_{\lambda} d \mu(\lambda) .
$$

Proof. Note first that

$$
\left|\left(\widehat{f}(\lambda), \pi_{\lambda}(x) s_{\lambda}\right)_{\lambda}\right| \leq\|\widehat{f}(\lambda)\|_{\lambda}\left\|\pi_{\lambda}(x) s_{\lambda}\right\|_{\lambda}=\|\widehat{f}(\lambda)\|_{\lambda}
$$

Using (8) we get

$$
\begin{aligned}
f(x) & =\ell\left(x^{-1}\right) f(e) \\
& =\int_{\Lambda}\left(\mathcal{F}\left(\ell\left(x^{-1}\right) f\right)(\lambda), s_{\lambda}\right)_{\lambda} d \mu(\lambda) \\
& =\int_{\Lambda}\left(\pi_{\lambda}\left(x^{-1}\right) \widehat{f}(\lambda), s_{\lambda}\right)_{\lambda} d \mu(\lambda) \\
& =\int_{\Lambda}\left(\widehat{f}(\lambda), \pi_{\lambda}(x) s_{\lambda}\right)_{\lambda} d \mu(\lambda) \\
& =\int_{\Lambda} \int_{G / K} f(y)\left(s_{\lambda}, \pi_{\lambda}\left(y^{-1} x\right) s_{\lambda}\right)_{\lambda} d y d \mu(\lambda) \\
& =\int_{\Lambda} f * \varphi_{\lambda}(x) d \mu(\lambda) .
\end{aligned}
$$

The rest follows now from (9).

Definition 4.11. Let $\Omega \subset \Lambda$ be measurable with finite measure. The function $f \in L^{2}(G / K)$ is called $\Omega$-bandlimited if $\operatorname{supp}(\widehat{f}) \subseteq \Omega$. We denote the space of $\Omega$-bandlimited functions by $L_{\Omega}^{2}(G / K)$. 
From now on we will always assume that $\Omega \subset \Lambda$ has finite measure. Define a section $\widehat{\psi} \in \int_{\Lambda}^{\oplus}\left(\pi_{\lambda}, \mathcal{H}_{\lambda}\right) d \mu(\lambda)$ by

$$
\widehat{\psi}(\lambda)=\chi_{\Omega}(\lambda) s_{\lambda}
$$

where $\chi_{\Omega}$ is the characteristic function on $\Omega$. Note that $\|\widehat{\psi}\|=\sqrt{\mu(\Omega)}<\infty$. Let $\psi:=$ $\mathcal{F}^{-1}(\widehat{\psi})$. Then $\psi \in L_{\Omega}^{2}(G / K)$ and $\|\psi\|_{2}=\sqrt{\mu(\Omega)}$. By Lemma 4.10 and the fact that $\overline{\varphi_{\lambda}\left(x^{-1}\right)}=\varphi_{\lambda}(x)$ we get:

Lemma 4.12. We have $\psi(x)=\int_{\Omega} \varphi_{\lambda}(x) d \mu(\lambda)$ and $\psi(x)=\overline{\psi\left(x^{-1}\right)}$.

Lemma 4.13. Let the notation be as above. Then the following holds:

(a) $L_{\Omega}^{2}(G / K)$ is a $G$-invariant Hilbert space.

(b) The orthogonal projection onto $L_{\Omega}^{2}(G / K)$ is given by $f \mapsto f * \psi$. In particular $\psi * \psi=\psi$ and $\psi$ is cyclic in $L_{\Omega}^{2}(G / K)$.

(c) $L_{\Omega}^{2}(G / K) \subset C(G / K)$.

(d) If $f \in L_{\Omega}^{2}(G / K)$ then $|f(x)| \leq \sqrt{\mu(\Omega)}\|f\|_{2}$. In particular, the point evaluation map $\mathrm{ev}_{x}: L^{2}(G / K) \rightarrow \mathbb{C}, f \mapsto f(x), x \in G / K$ is continuous.

Proof. (国) and (b) follow from Lemma $4.9 \mathcal{F}(\ell(x) f)=\pi_{\lambda}(x) \widehat{f}(\lambda)$ and

$$
\mathcal{F}(f * \psi)(\lambda)=\pi_{\lambda}(f) \mathcal{F}(\psi)(\lambda)=\chi_{\Omega}(\lambda) \pi_{\lambda}(f) s_{\lambda}=\chi_{\Omega}(\lambda) \mathcal{F}(f)(\lambda) .
$$

For (드) we note that if $f \in L_{\Omega}^{2}(G / K)$ then $f=f * \psi$ and hence $f$ is continuous. It follows that the evaluation map $\mathrm{ev}_{x}, x \in G / K$ is well defined. Furthermore, $|f(x)|=|f * \psi(x)| \leq$ $\|f\|_{2}\|\psi\|_{2}$ and (d) follows.

Remark 4.14. This shows that the spaces of bandlimited functions are natural spaces to study sampling. In particular each of those spaces is a reproducing kernel Hilbert space with reproducing kernel $K(x, y)=\overline{\psi\left(y^{-1} x\right)}$.

Theorem 4.15. Let $\Omega \subset \Lambda$ be measurable with finite measure. Let $S=L_{\Omega}^{2}(G / K)$. Then $L_{\Omega}^{2}(G / K)=\operatorname{Co}_{S}^{\psi}\left(L^{2}(G / K)\right)$ is a Coorbit space.

Proof. We have for $f \in L^{2}(G / K)$

$$
V_{\psi}(f)(x)=(f, \lambda(x) \psi)=f * \psi(x) .
$$

The claim follows therefore from Lemma 4.13.

Remark 4.16. If $G$ is a Lie group, then we can replace $S=L_{\Omega}^{2}(G / K)$ by the space $S=$ $L_{\Omega}^{2}(G / K)^{\infty}$ of smooth vectors, i.e., all $f \in L_{\Omega}^{2}(G / K)$ such that $D f \in L_{\Omega}^{2}(G / K)$ for all invariant differential operators $D$.

Remark 4.17. If we can choose a topology on $\Lambda$ such that $\lambda \mapsto \pi_{\lambda}(x) s_{\lambda}$ is continuous and $\Omega$ is chosen to be compact, then the function $\psi$ cannot be integrable. If it were this would mean that $\widehat{\psi}$ is continuous, which is not the case. Therefore we have another example for which integrability is not necessary.

Example 4.18 ( $G$ abelian). If $G$ is abelian then

$$
L^{2}(G) \simeq L^{2}(\widehat{G}, d \mu) \simeq \int_{\widehat{G}}^{\oplus}(\chi, \mathbb{C}) d \mu(\chi)
$$


where $\widehat{G}$ is the dual group consisting of all continuous characters $\chi: G \rightarrow \mathbb{T}$ and $d \mu$ is the dual Haar measure on $\widehat{G}$. In this case we can choose $s_{\chi}=1$ for all $\chi \in \widehat{G}$. The Fourier transform is given by

$$
\widehat{f}(\chi)=\int_{G} f(x) \chi(x) d x .
$$

Note, that the usual conjugation is missing. The function $\psi$ is

$$
\psi(x)=\int_{\Omega} \overline{\chi(x)} d \mu(\chi)=\int_{\Omega} \chi\left(x^{-1}\right) d \mu(\chi) .
$$

If $G=\mathbb{R}$ and $\Omega=[-R / 2, R / 2]$ then

$$
\psi(x)=\int_{-R / 2}^{R / 2} e^{-2 \pi i \lambda x} d \lambda=\frac{\sin (R \pi x)}{\pi x}=R \operatorname{sinc}(R \pi x)
$$

as usually. The Shannon sampling theorem is then

$$
f(x)=\sum_{n=-\infty}^{\infty} f(n / R) \frac{\sin (R \pi(x-n / R)}{\pi(x-n / R)} .
$$

In general, if there exists a discrete subgroup $\widehat{\Gamma} \subset \widehat{G}$ such that $\Omega \simeq \widehat{G} / \widehat{\Gamma}$, ie, $\Omega+\widehat{G}$ is a measurable tiling of $\widehat{G}$ then we have with the same proof as in the classical case:

$$
f(y)=\sum_{x \in \widehat{\Gamma}^{\perp}} f(x) \psi\left(x^{-1} y\right)
$$

uniformly. Here

$$
\widehat{\Gamma}^{\perp}=\{x \in G \mid(\forall \gamma \in \widehat{\Gamma}) \gamma(x)=1\} .
$$

The interesting case is therefore the irregular sampling. That discussion has been started in [7].

Example 4.19 (Compact groups). Let $K$ be a compact group. Set $G=K \times K$ and identify $K$ with the diagonal subgroup $K \simeq\{(k, k) \in G \mid k \in K\}$. The space $G / K$ can be identified with $K$ by $(a, b) K \mapsto a b^{-1}$. The left regular action on $G / K$ is then transformed to the left-right action of $K$ on $L^{2}(K)$, i.e., $\ell_{K} \otimes r_{K}(a, b) f(x)=f\left(a^{-1} x b\right)$. For an irreducible representation $\left(\pi, V_{\pi}\right)$ of $K$ define a representation of $G$ on $\operatorname{End}\left(V_{\pi}\right)$ by

$$
\widetilde{\pi}(a, b) T=\pi(a) \circ T \circ \pi\left(b^{-1}\right) .
$$

Then

$$
L^{2}(K)=L^{2}(G / K) \simeq \bigoplus_{\pi \in \widehat{G}}\left(\tilde{\pi}, \operatorname{End}\left(V_{\pi}\right)\right) .
$$

We choose $s_{\pi}=\mathrm{I}_{V_{\pi}}$. Then $\widehat{f}(\pi)=\pi\left(f^{\vee}\right) I_{V_{\pi}}$ where $f^{\vee}(x)=f\left(x^{-1}\right)$. The inversion formula reads

$$
f(x)=\sum_{\pi} d(\pi) \operatorname{Tr}(\pi(x) \widehat{f}(\pi)) .
$$

A subset $\Omega \subset \widehat{G}$ is of finite measure if and only if $\Omega$ is finite. In that case we have

$$
\psi(x)=\sum_{\pi \in \Omega} d(\pi) \operatorname{Tr}\left(\pi(x) I_{V_{\pi}}\right)
$$


and $\widehat{\psi}(\pi)=I_{V_{\pi}}$. Furthermore

$$
L_{\Omega}^{2}(G / K)=\left\{\sum_{\pi \in \Omega} d(\pi) \operatorname{Tr}\left(\pi(x) T_{\pi}\right) \mid(\forall \pi \in \Omega) T_{\pi} \in \operatorname{End}\left(V_{\pi}\right)\right\} .
$$

Example 4.20 (Riemannian symmetric spaces of the noncompact type). The last example we would like to mention is the case of Riemannian symmetric spaces $G / K$ of the noncompact type, which always comes from a Gelfand pair $(G, K)$. We will not describe this case here in detail as it would require us to introduce several pages of new notation. We would however like to point out the article by Pesenson [17] where irregular sampling is discussed. Note, that according to [14], see also [16] every function $f$ in $L_{\Omega}^{2}(G / K)$ extends to a holomorphic function on a complex neighborhood of $G / K$ and hence $f$ is determined on any infinite set with a limit point.

\section{REFERENCES}

[1] H. Chébli and J. Faraut. Fonctions holomorphes à croissance modérée et vecteurs distributions. Math. Z., 248(3):540-565, 2004.

[2] J. Christensen and G. Ólafsson. Coorbit spaces dual pairs. To be submitted.

[3] M. Duflo and C. C. Moore. On the regular representation of a nonunimodular locally compact group. J. Functional Analysis, 21(2):209-243, 1976.

[4] H. G. Feichtinger and K. H. Gröchenig. A unified approach to atomic decompositions via integrable group representations. In Function spaces and applications (Lund, 1986), volume 1302 of Lecture Notes in Math., pages 52-73. Springer, Berlin, 1988.

[5] H. G. Feichtinger and K. H. Gröchenig. Banach spaces related to integrable group representations and their atomic decompositions. I. J. Funct. Anal., 86(2):307-340, 1989.

[6] H. G. Feichtinger and K. H. Gröchenig. Banach spaces related to integrable group representations and their atomic decompositions. II. Monatsh. Math., 108(2-3):129-148, 1989.

[7] H. G. Feichtinger and K. H. Gröchenig. Irregular sampling theorems and series expansions of bandlimited functions. J. Math. Anal. Appl., 167(2):530-556, 1992.

[8] H. G. Feichtinger and S. S. Pandey. Recovery of band-limited functions on locally compact Abelian groups from irregular samples. Czechoslovak Math. J., 53(128)(2):249-264, 2003.

[9] K. H. Gröchenig. Describing functions: atomic decompositions versus frames. Monatsh. Math., 112(1):142, 1991.

[10] Harish-Chandra. Representations of semisimple Lie groups. IV. Amer. J. Math., 77:743-777, 1955.

[11] Harish-Chandra. Representations of semisimple Lie groups. V. Amer. J. Math., 78:1-41, 1956.

[12] M. Holschneider. Wavelets. Oxford Mathematical Monographs. The Clarendon Press Oxford University Press, New York, 1995. An analysis tool, Oxford Science Publications.

[13] A. W. Knapp. Representation Theory of Semisimple Groups. Princeton Landmarks in Mathematics. Princeton University Press, Princeton, NJ, 2001. An overview based on examples, Reprint of the 1986 original.

[14] B. Krötz and R. J. Stanton. Holomorphic extensions of representations. II. Geometry and harmonic analysis. Geom. Funct. Anal., 15(1):190-245, 2005.

[15] G. Ólafsson and B. Ørsted. The holomorphic discrete series for affine symmetric spaces. I. J. Funct. Anal., 81(1):126-159, 1988.

[16] G. Ólafsson and H. Schlichtkrull. Representation theory, Radon transform and the heat equation on a Riemannian symmetric space. To appear in a special volume of Contemp. Math. in honor of G. Mackey., 2007.

[17] I. Pesenson. A discrete Helgason-Fourier transform for Sobolev and Besov functions on noncompact symmtric spaces. In Radon Transforms, Geometry, and Wavelets, volume 464, pages 231-247. Amer. Math. Soc., Providence, RI, 2008.

[18] H. Rauhut. Time-Frequency and Wavelet Analysis of Functions with Symmetry Properties. LogosVerlag, 2005. 
[19] H. Triebel. Function spaces on Lie groups, the Riemannian approach. J. London Math. Soc. (2), 35(2):327-338, 1987.

[20] H. Triebel. Characterizations of Besov-Hardy-Sobolev spaces: a unified approach. J. Approx. Theory, $52(2): 162-203,1988$.

[21] G. Warner. Harmonic Analysis on Semi-Simple Lie Groups. I. Springer-Verlag, New York, 1972. Die Grundlehren der mathematischen Wissenschaften, Band 188.

[22] J. A. Wolf. Harmonic Analysis on Commutative Spaces, volume 142 of Mathematical Surveys and Monographs. American Mathematical Society, Providence, RI, 2007.

[23] G. Zimmermann. Coherent states from nonunitary representations. In Topics in multivariate approximation and interpolation, volume 12, pages 231-247. Elsevier, Amsterdam, 2005.

329 Lockett Hall, Department of mathematics, Louisiana State University

E-mail address: vepjan@math.lsu.edu

$U R L:$ http://www.math.1su.edu/ ${ }^{\text {vepjan }}$

322 Lockett Hall, Department of mathematics, Louisiana State University

E-mail address: olafsson@math.lsu.edu

URL: http://www.math.lsu.edu/ olafsson 